\title{
Moving Live Dissociated Neurons With an Optical Tweezer
}

\author{
Jerome Pine* and Gary Chow
}

\begin{abstract}
The use of an optical tweezer for moving dissociated neurons was studied. The main features of the tweezers are outlined as well as the general principles of its operation. Infrared beams at 980 and $1064 \mathrm{~nm}$ were used, focused so as to make a trap for holding neurons and moving them. Absorption by cells at those wavelengths is very small. Experiments were done to evaluate nonsticky substrate coatings, from which neurons could be easily lifted with the tweezers. The maximum speed of cell movement as a function of laser power was determined. Detailed studies of the damage to cells as a function of beam intensity and time of exposure were made. The $980 \mathrm{~nm}$ beam was much less destructive, for reasons that are not understood, and could be used to safely move cells through distances of millimeters in times of seconds. An illustrative application of the use of the tweezers to load neurons without damage into plastic cages on a glass substrate was presented. The conclusion is that optical tweezers are an accessible and practical tool for helping to establish neuron cultures of cells placed in specific locations.
\end{abstract}

Index Terms-Cultured neurons, multielctrode array, optical tweezers.

\section{INTRODUCTION}

I N 1970, Arthur Ashkin discovered the utility of a focused laser beam for manipulating micrometer-sized transparent particles [1]. During the years since then, a multiplicity of applications evolved from this beginning, and the history and development of applications during the decades afterward is summarized in a review he wrote [2]. A key development was the realization in 1986 that a focused laser beam could trap and move a refractile particle in three dimensions [3]. Fig. 1 qualitatively illustrates the general principle. At left, a net lateral force from refraction moves a spherical bead to the higher intensity at the centerline of the beam. At right, the refraction of the focused beam produces a net force upward along the beam direction to hold it near the focus. As the figure suggests, the longitudinal force can be toward the incoming beam, and it can overcome the recoil force due to beam reflection (not shown) as well as a gravitational downward force. The particle is held closely centered on the beam focus, hence the term "optical tweezers." A key ingredient for making the tweezers work is that the focused laser

Manuscript received May 23, 2008; revised August 14, 2008. First published October 31, 2008; current version published May 6, 2009. This work was supported in part by the National Institutes of Health under Grant NS-044134. Asterisk indicates corresponding author.

*J. Pine is with California Institute of Technology, Pasadena, CA 91125 USA (e-mail: jpmail@capsi.caltech.edu).

G. Chow is with California Institute of Technology, Pasadena, CA 91125 USA (e-mail: gangc@its.caltech.edu).

Color versions of one or more of the figures in this paper are available online at http://ieeexplore.org.

Digital Object Identifier 10.1109/TBME.2008.2005641
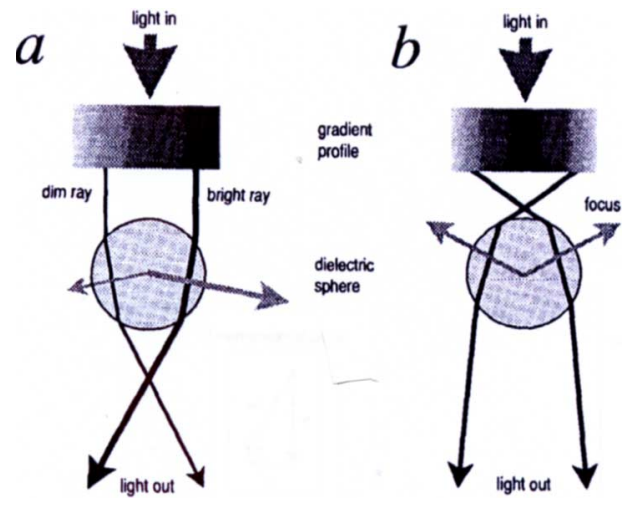

Fig. 1. Tweezer forces. (From Svoboda and Block, Ann. Rev. Biophys, Biomol. Struct., v. 23, p. 250, 1994).

beam subtends a wide angle, from a lens of large numerical aperture. Typically, oil immersion objective lenses with numerical apertures greater than 1.25 , and cone half-angles greater than $55^{\circ}$, are needed. The short working distances of such lenses needs to be considered in applications of the tweezers.

Our study is an example with a general application to using tweezers to lift, carry, and place single neurons at specific locations in a cell culture dish. In fact, there has been in recent years considerable interest in growing cultured neurons that are placed in controlled locations rather than randomly in a culture dish [4], [5]. An important example is that of Townes-Anderson and her colleagues [6], who used optical tweezers to place different types of retinal cells next to each other in culture to study their interactions. This is the earliest study using tweezers and whole neurons, and was done with a proprietary turnkey system for which no detailed data are available. The major issue is possible damage to the cells, and dependence on laser characteristics was not examined in detail. Other whole neuron experiments have more recently been done, without damage studies [7], [8].

We were motivated by the need to place neurons quickly and precisely into parylene plastic "cages" about $30 \mu \mathrm{m}$ in diameter, in an array on a culture dish, without injuring them. Fig. 2 shows a scanning electron micrograph of a cage, fabricated in the Tai laboratory at Caltech by Angela Tooker [9]. A neuron that is placed in it attaches to the bottom near an electrode, which can be used for extracellular stimulation or recording. It can grow axons and dendrites out through any of the six radial tunnels, which are $1 \mu \mathrm{m}$ high and $10 \mu \mathrm{m}$ wide, too small to allow escape through them. An array of these cages with neurons in them, spaced about $100 \mu \mathrm{m}$ apart, will support the growth and development of a cultured network. The fact that the neurons are held near the electrodes provides the opportunity to 


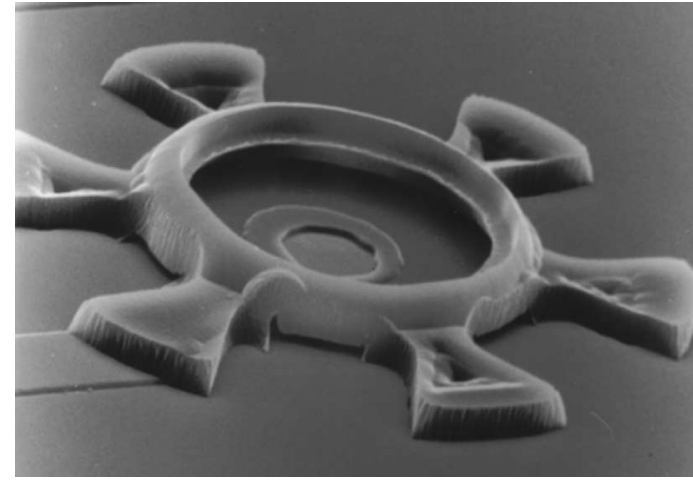

Fig. 2. Parylene cage. The electrode at the bottom is gold and will be platinized for lower impedance. There are six tunnels for outgrowth and six anchors to hold it in place.

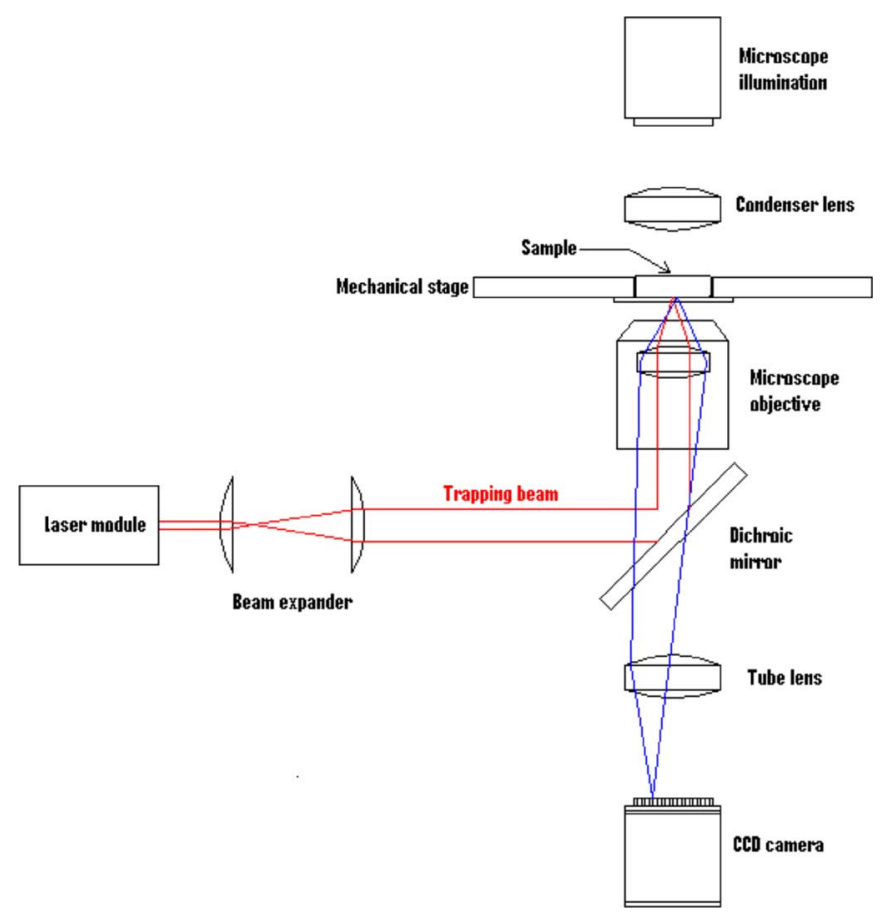

Fig. 3. Schematic view of the tweezers optical system.

monitor the growth and the changing connectivity over time by electrical stimulation and recording. The ability to do this for weeks provides a unique opportunity for studying networks.

\section{Materials AND MEthodS}

\section{A. Optical System}

A schematic view of the main components of a tweezer is shown in Fig. 3, for an inverted microscope. The main features are the laser beam optics, including a large numerical aperture oil-immersion objective, standard phase contrast microscope illumination, and a charge-coupled device video camera that can view a phase contrast or bright field image through the dichroic IR mirror. It can also simultaneously see the reflection of the laser spot off the coverslip-culture medium interface. The narrow laser beam needs to fill the back aperture of the objective in order to maximize the angular spread of the focused beam, and a $5 \times$ beam expander (Thorlabs BE05) is used. The culture dish is moved by a mechanical stage that has micrometer resolution, and moves it with respect to the neuron held stationary in the tweezer beam. The beam was aligned with mirrors before the expander when a 1-W 1064-nm YAG laser was used (Intelite ISF064-1000P). When a 500-mW diode laser (JDS Uniphase) was used, the diode was provided matched to a flexible singlemode optical fiber and this was attached to a fiber collimator (Microlaser FC5) mounted just before the beam expander. No mirror steering was needed.

The stage (ASI M2000) was controllable through a serial port from a PC, and LabView software was written to carry a neuron from any initial location to a selected cage location, utilizing the capabilities of the stage and its controller. The stage moves in all three dimensions.

\section{B. Objective}

The transmission of large numerical aperture objectives is not well specified at $1064 \mathrm{~nm}$ [10]. A simple measure of the transmission could be made if a detector at the lens focus was efficient over the large range of incident angles of the focused light. We measured the angular dependence of the sensitivity of a standard broadband silicon diode IR power detector (Coherent PM10-19B), and it was uniformly high up to an incident angle of $75^{\circ}$. It was used to measure the transmission at $1064 \mathrm{~nm}$ of a Zeiss neofluor objective (\#440460), n.a. 1.25, 63×, and the result, $62 \%$, was in accord with the Zeiss specification of $60 \%$. This objective was used in our experiments. A competitive lens with transmission specified as $40 \%$ was found to transmit only $18 \%$. The power detector was suitable for measuring the incident beam at the image position on the mechanical stage, so that the power incident on a neuron at the focus was reliably determined, and reported with the damage data.

\section{The Nonstick Substrate}

The cultures that we used in our experiments were grown on thin glass cover slips, thickness about $100 \mu \mathrm{m}$, because of the short working distance of the objective. After lifting, we wanted to be able to carry the neuron far enough above the cover slip to ameliorate the effects of passing over opaque gold conductors connected to the electrodes of the array. A beam focused $100 \mu \mathrm{m}$ above the $10-\mu \mathrm{m}$-wide conductors would not be significantly blocked, nor would it vaporize the conductors. We determined that newly dissociated neurons would attach to an uncoated cover slip so that within minutes the cells could not be lifted by the tweezers. This led to a series of studies of various coatings for the cover slip and of pretreatments of the neurons. The best pretreatment was with trypsin, an enzyme used in cell dissociation, which made the cell membrane less sticky and provided about 30 min during which neurons could be lifted reliably. However, cells treated this way had very poor survival after moving them and plating them on our standard coating. A large number of purported nonsticky coatings were tried. Teflon was useless, as were a variety of silanes with polyethylene glycol attached, which provided $10 \mathrm{~min}$ or less of 


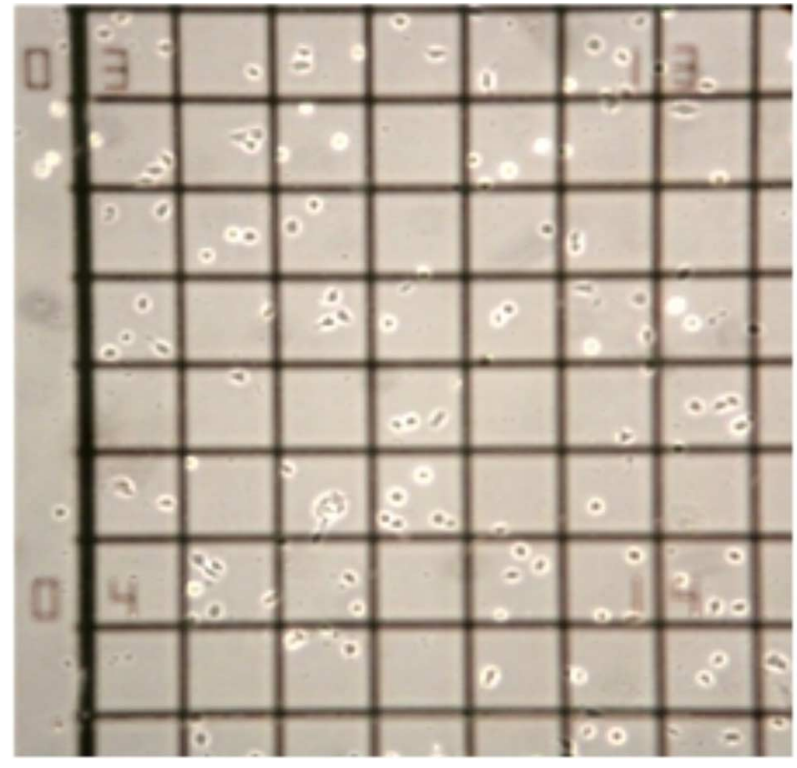

Fig. 4. Culture dish locating grid, with 2-h old neurons plated on it. Labels of major squares, with $6 \times 6$ small squares, can be seen.

lifting time. Bovine serum albumin (BSA) was good for $30 \mathrm{~min}$ but optically rough. The best coating, and the one we have used, was "poly(2-hydroxyethyl methacrylate (poly-HEMA)," Sigma P-3932). Applied in several thin layers, it is optically smooth and preserves its nonstickiness for more than $1 \mathrm{~h}$.

\section{Cell Culture}

Neurons were dissected from the hippocampi of rat embryos at 18 days of gestation. The hippocampal tissue was diced and immersed in $0.25 \%$ trypsin (Sigma T1426) at $37^{\circ} \mathrm{C}$. for $15 \mathrm{~min}$, then rinsed in a medium with serum to neutralize the trypsin, and triturated through a pipette tip. The solution was spun for 6 min to separate the cells to the bottom of the tube in a layer of BSA. Cells were then resuspended in Hank's balanced salt solution and stored at $4{ }^{\circ} \mathrm{C}$ until used.

The culture dish was a $35-\mathrm{mm}$-diameter polystyrene plastic dish with a $10-\mathrm{mm}$-diameter hole at the center, under which a number 0 cover slip was glued, and on which cells were grown. Cells to be lifted were gently deposited on a poly-HEMA-coated region of the culture dish. The remaining area was treated with $0.05 \%$ polyethyleneimine (Sigma P3143) in borate buffer for several hours and then rinsed and dried. Dissociated neurons deposited on this area adhered in minutes and grew well for weeks. A cell-locating grid of gold lines $10 \mu \mathrm{m}$ wide in labeled $100 \mu \mathrm{m}$ squares was deposited on the cover slip in the area on which cells were grown, shown in Fig. 4. The culture medium was Neurobasal (Gibco 21103-049) with 2\% B27 (Gibco 17504044), 0.25\% Glutamax (Gibco 35050-079), and 5\% horse serum (Hyclone). The cells were incubated at $35.5^{\circ} \mathrm{C}$ in an atmosphere of air plus $5 \% \mathrm{CO}_{2}$. They were fed every seven days with $50 \%$ fresh medium after withdrawing 50\%.

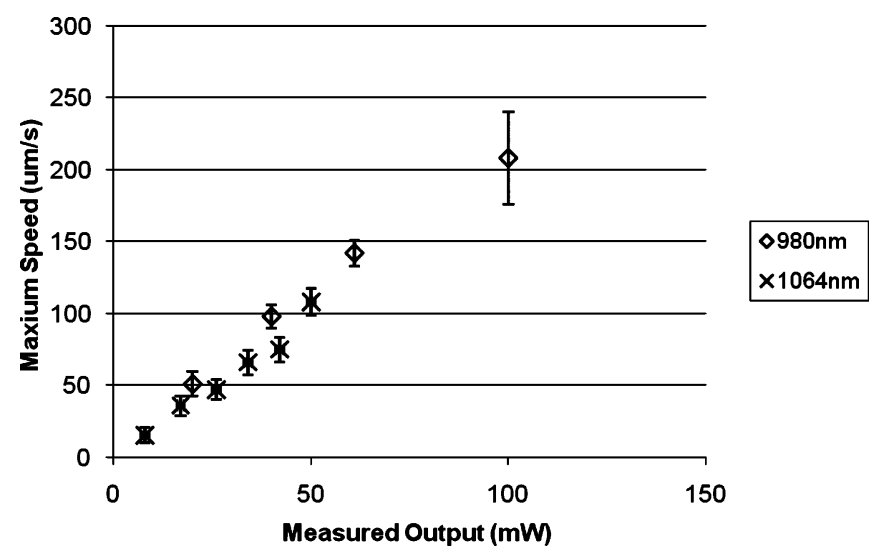

Fig. 5. Maximum speed versus laser power.

\section{RESULTS}

\section{A. Speed Versus Laser Power}

With poly-HEMA, the power needed to lift freshly dissected hippocampal cells $10-15 \mu \mathrm{m}$ in diameter was only about $20 \mathrm{~mW}$. However, it was important to learn the speed with which a lifted cell can be transported (motion of stage, with laser beam fixed). In our application, the planned motion was up to $5 \mathrm{~mm}$, and the goal was to move 60 neurons in less than an hour. Allowing half the time to identify and lift cells and half for a computer-controlled move to a selected cage, the speed required would be about $170 \mu \mathrm{m}$ per second. At a given beam power, cells were lifted and carried at increasing speed until they were lost. Twelve typical cells were tested at each power. The results for the mean maximum speed and the standard error of the mean are shown in Fig. 5. They indicate that the speed increases linearly with beam power, and that, as might be expected, there is no significant difference between the $1064 \mathrm{~nm}$ laser and the $980 \mathrm{~nm}$ laser. There is a need for laser power at the focus of about $80 \mathrm{~mW}$ in order to meet the design goal.

Ray-optics-based calculations of the trapping force on polystyrene spheres have been made by Ashkin [10] and indicate that the force is proportional to beam power, as shown in the figure, and independent of sphere size. The linear dependence of maximum speed on power is expected from the fact that the drag is linearly proportional to velocity in this Reynolds Number regime.

\section{B. Irradiation Damage Studies Without Cages}

1) Stationary Cells at $1064 \mathrm{~nm}$ (Effect of Laser Power): Studies were made on freshly dissected stationary cells plated on the culture dish grid, on which the laser beam was focused at a chosen power for varying lengths of time. It was seen that cells that grew for three days developed normally for many days afterward, identically to control nonirradiated cells, so survival data were typically obtained at days 1,2 , and 3 . The procedure used was to record on a list the locations on the grid of a population of healthy looking cells, usually $40,2 \mathrm{~h}$ after plating, and to randomly select ten cells for irradiation. Typically, 20 chosen randomly from the remainder were used as "control" 


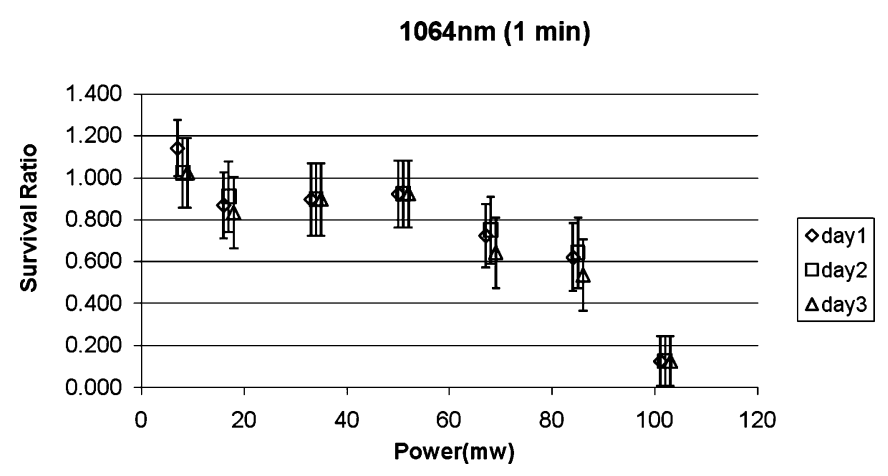

Fig. 6. Survival ratio versus laser power at $1064 \mathrm{~nm}$ for 1 min exposure of freshly plated neurons. Data from 83 irradiated neurons and 270 control neurons.

cells. Data were combined from two or more experiments, and glial cells, which became identifiable after a day, as well as the few cells that disappeared, were removed from the initial populations. The survival ratio is the probability of survival of the irradiated cells divided by that of the nonirradiated control cells. Ratios for a 1 min exposure to the $1064 \mathrm{~nm}$ laser at varying power are shown in Fig. 6. The survival probabilities for both cell populations reflect a binomial distribution, from which their uncertainties are determined. Control cells also have significant cell death during the three-day period. Dead neurons are clearly identifiable because they shrivel up and the skeletons remain. For both irradiated and control populations. the survival probability at a given day is found from the number of live neurons divided by the corrected number of initial neurons. The errors are not independent for each day, but come from uncertainties in the number of each population based on a binomial distribution. The data points on the graph directly indicate the ratio of the cell numbers. The ratio can be greater than 1, depending on the chance rates of death of irradiated and control cells. The uncertainty in the ratio is propagated from those of the two individual survival probabilities, combined in quadrature. It is quite significant, largely due to the deaths by day 1 .

The data show a dramatic increase in death rate for powers greater than $50 \mathrm{~mW}$. It is also clear at all powers that if neurons survive after one day, they almost always remain viable for three days, a time after which we find no long-term effects. The damage is clearly an acute effect, and it seems able to heal for some neurons. The maximum safe power of $50 \mathrm{~mW}$ is somewhat less than the $80 \mathrm{~mW}$ for the design speed goal.

2) Stationary Cells at $980 \mathrm{~nm}$ (Effect of Laser Power): Using the same procedure as discussed for $1064 \mathrm{~nm}$, the results for the $980 \mathrm{~nm}$ laser are shown in Fig. 7. Data were collected up to day 4. It can be seen that the survival was excellent to at least twice the power level of the $1064 \mathrm{~nm}$ laser, and the loss at $130 \mathrm{~mW}$ is not statistically significant. The big difference between the two wavelengths is surprising, since there is no obvious damage mechanism-such as membrane permeabilization or heating - that should be so sensitive to wavelength. However, previous studies of damage to E. coli cells and reduction of replication rate of Chinese hamster ovary $(\mathrm{CHO})$ cells show a similar wavelength dependence [11], [12]. The heating has been studied by Liu et al. [13], and found to be unimportant for us,

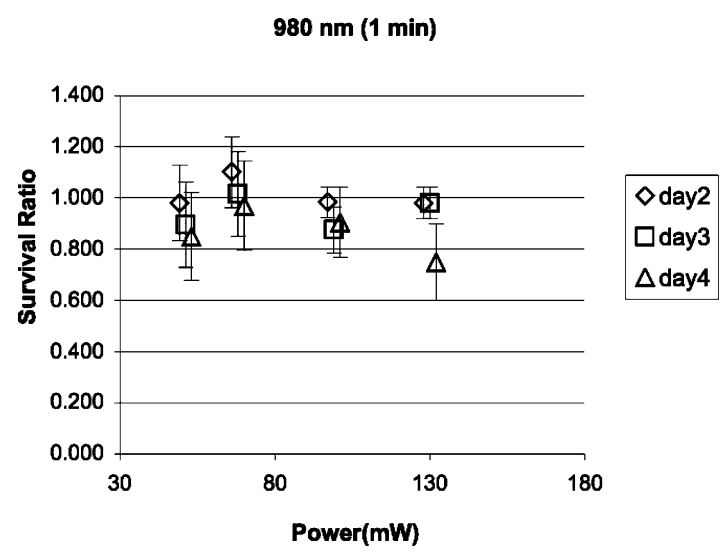

Fig. 7. Survival versus laser power at $980 \mathrm{~nm}$ for a 1 min exposure of freshly plated neurons. Data from 76 irradiated neurons and 210 control neurons.

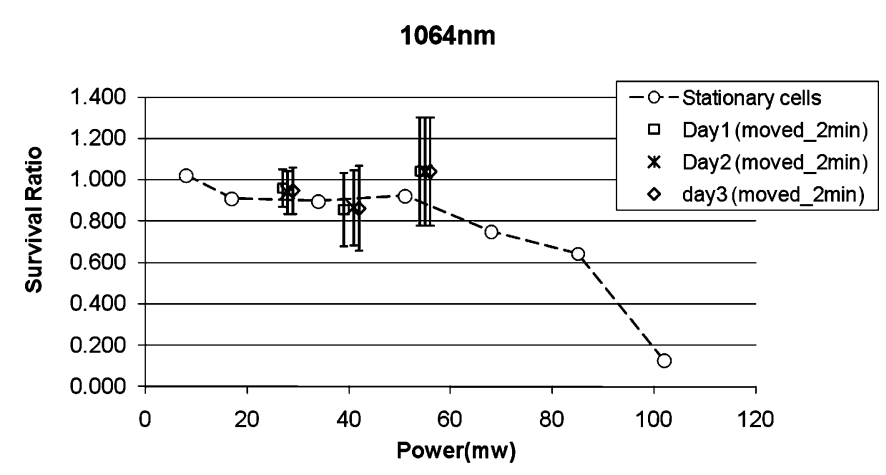

Fig. 8. Survival of moved cells at $1064 \mathrm{~nm}$, compared with stationary cells. Moved data from 88 irradiated neurons and 80 control neurons.

about $1^{\circ}$ per $100 \mathrm{~mW}$ at $1064 \mathrm{~nm}$ in $\mathrm{CHO}$ cells. The difference remains a mystery and has not been explained by any of those who have previously seen it.

3) Moved Cells (Effect of Laser Power): The logistics of the tweezers system development made it attractive to start early with experiments on stationary cells. However, to be realistic, cells needed to be picked up, held, carried by the tweezers, and dropped onto a polyetherimide (PEI) substrate for subsequent growth. The spatial relationship between the beam and the neuron could be somewhat different than when the neuron was anchored to the substrate, although stationary cells were irradiated before they flattened and grew processes. Fig. 8 shows, for the $1064 \mathrm{~nm}$ laser, a comparison of the survival ratio versus power for moved neurons held for $2 \mathrm{~min}$. The laser at that time could not deliver more than $51 \mathrm{~mW}$ to the focal spot. The comparison does not indicate a significant difference for the moved cells in comparison with the stationary cells irradiated for $1 \mathrm{~min}$. At $980 \mathrm{~nm}$, a single extreme condition, $4 \mathrm{~min}$ at $130 \mathrm{~mW}$, was compared with results for stationary cells. The survival ratio was $1.0 \pm 0.1 \mathrm{in}$ both cases.

\section{Damage Studies for Caged Neurons}

The final test was to use the tweezers to move neurons into cages and observe their survival. At $980 \mathrm{~nm}$, the power was 


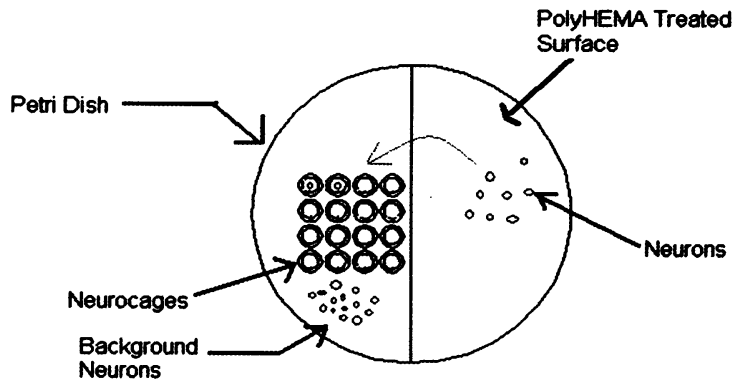

Fig. 9. Trial cell culturing with cages (not to scale).

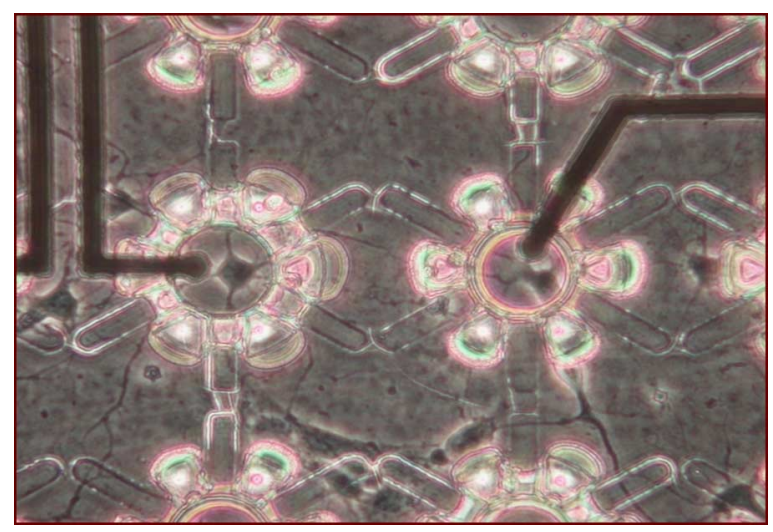

Fig. 10. Neurons in cages at three days in vitro.

$130 \mathrm{~mW}$, and the time was $2 \mathrm{~min}$, more than sufficient for the design goal. Fig. 9 next schematically summarizes a procedure alluded to previously. Approximately half the cover slip area was treated with poly-HEMA and the other half with PEI for cell attachment. About 20,000 background neurons were grown to condition the medium, and then plated and attached first in an area away from the cages. Neurons were then deposited on the poly-HEMA, lifted with the tweezers, and deposited in cages that were attached to the cover slip. Fig. 10 shows a view of two of the cages in the array containing 3-day-old neurons with dark cell bodies and processes, growing in them and out through their tunnels. Control neurons, outside the cages but in among them, carried from the source of the lifted cells by currents in the dish, are seen at the left and right sides of the figure. The survival ratio of cells moved into cages compared with background cells was on average $95 \%$, with $10 \%$ uncertainty, for 1-10 days in vitro.

\section{CONCLUSION}

It has been found that dissociated neurons can be easily picked up and carried with laser tweezers if they are on a poly-HEMAtreated surface. They can be moved at speeds of $200 \mu \mathrm{m} / \mathrm{s}$ or more by IR tweezers with an intensity of $100 \mathrm{~mW}$ at the sample. The tweezer apparatus involves relatively inexpensive additions to an inverted microscope with a high-magnification wide-aperture lens. Experiments to evaluate damage to neurons by the laser beam showed that $980 \mathrm{~nm}$ radiation is much less damaging than $1064 \mathrm{~nm}$ radiation, but no model has been found by ourselves or others that explains the difference. The $500-\mathrm{mW}$ 980-nm diode laser used is inexpensive, long-lived, and easy to connect to a beam expander illuminating the epi-illumination fluorescence port of the microscope. An example in which the tweezers were used to load neurons into cages for forming cultured networks provided an illustration of the possibilities of using tweezers in experiments with cultured cells placed at specific locations.

\section{REFERENCES}

[1] A. Ashkin, "Acceleration and trapping of particles by radiation pressure," Phys. Rev. Lett., vol. 24, pp. 156-159, 1970.

[2] A. Ashkin, "Optical trapping and manipulation of neutral particles using lasers," in Proc. Natl. Acad. Sci., 1997, vol. 94, pp. 4853-4860.

[3] A Ashkin, J. M. Dziedzic, S. Chu, and J. E. Bjorkholm, "Observation of a single beam gradient force optical trap for dielectric particles," Opt. Lett., vol. 11, pp. 288-290, 1986.

[4] P. Fromherz, "Neuron-semiconductor chip with chemical synapse between identified neurons," Phy. Rev. Lett., vol. 92, pp. 038102-1-0381024, 2004.

[5] Y. Nam, J. C. Chang, B. C. Wheeler, and G. J. Brewer, "Gold-coated microelectrode array with thiol-linked self-assembled monolayers for engineering neuronal cultures," IEEE Trans. Biomed. Eng., vol. 51, no. 1, pp. 158-165, Jan. 2004.

[6] E. Townes-Anderson, R. S. St. Jules, D. M. Sherry, D. M. Lichtenberger, and M. Hassanain, "Micromanipulation of retinal neurons by optical tweezers," Mol. Vis., vol. 4, pp. 12-20, 1998.

[7] R. K. Pirlo, D. M. D. Dean, D. R. Knapp, and B. Z Gao, "Cell deposition system based on laser guidance," Biotechnol. J., vol. 1, pp. 1007-1013, 2006.

[8] R. J. Clarke, K. Hognason, M. Brimacombe, and E. Townes-Anderson, "Cone and rod cells have different target preferences in vitro as revealed by optical tweezers," Mol. Vis., vol. 14, pp. 706-720, 2008.

[9] A. Tooker, J. Meng, J. Erickson, Y-C. Tai, and J. Pine, "Biocompatible parylene neurocages," IEEE Eng. Med. Biol., vol. 24, no. 6, pp. 30-33, Nov./Dec. 2005.

[10] A. Ashkin, "Forces of a single-beam gradient laser trap on a dielectric sphere in the ray optics regime," Biophys. J., vol. 61, pp. 569-582, 1992.

[11] K. C. Neuman, E. H. Chadd, G. F. Liou, K. Bergman, and S. M. Block, "Characterization of photodamage to Escherichia coli in optical traps," Biophys. J., vol. 77, pp. 2856-2863, 1999.

[12] H. Liang, K. T. Vu, P. Krishman, T. C. Trang, D. Shin, S. Kimel, and M. W. Berns, "Wavelength dependence of cell cloning efficiency after optical trapping," Biophys. J., vol. 70, pp. 1529-1533, 1996.

[13] Y. Liu, G. J. Sonek, M. W. Berns, and C. F. Chapman, "Evidence for localized cell heating induced by infrared optical tweezers," Biophys. J., vol. 68, pp. 2137-2144, 1995.

Authors' photographs and biographies not available at the time of publication. 